\title{
Symmetrical interdigital hyperkeratosis of the hands
}

\section{Kwon Osung, Chung Hyun, Park Joonsoo}

\author{
Department of Dermatology, School of Medicine, Catholic University of Daegu, Namgu, Daegu, South Korea \\ Corresponding author: Joonsoo Park, M.D., Ph.D., E-mail: g9563009@cu.ac.kr
}

Sir,

Symmetrical interdigital hyperkeratosis is a rare disorder described by Frei in 1923 [1]. He reported a case of a patient with congenital circumscribed hyperkeratosis of the interdigital spaces of the hands and feet. There was a slight hyperkeratosis on the dorsal aspects of some interphalangeal joints and on the elbows and knees. No keratoderma or hyperhydrosis of the palms and soles was observed. Frei named this condition "Congenital Symmetrical Interdigital Hyperkeratosis”.

A 35-year-old man presented with a symmetrical keratoderma localized to the interdigital spaces of the fingers. Skin thickening was developed when he was 15 years old. There was no relevant family history. There were no occupational and traumatic factors those could have affected this condition. There were no symptoms or signs of preceding infections. On physical examination, there were dark brownish colored hyperkeratotic plaques on his both first, second, third interdigital spaces of hands and the lesions slightly extended over dorsal aspects of some metacarpophalangeal joints of hands (Fig. 1). At the palm, there were no keratoderma and hyperhidrosis. The finger nails were normal. Skin biopsy showed orthokeratotic hyperkeratosis, hypergranulosis, acanthosis (Fig. 2). Although he was treated with keratolytics, there was no response to keratolytics. Clinical and histological features were consistent with the diagnosis of symmetrical interdigital hyperkeratosis.

Since the first case was reported in 1923 , few cases have been reported in the literatures. In 1990 , Salamon reported a similar condition in a 19-yearold male [2]. The skin lesions were limited to the

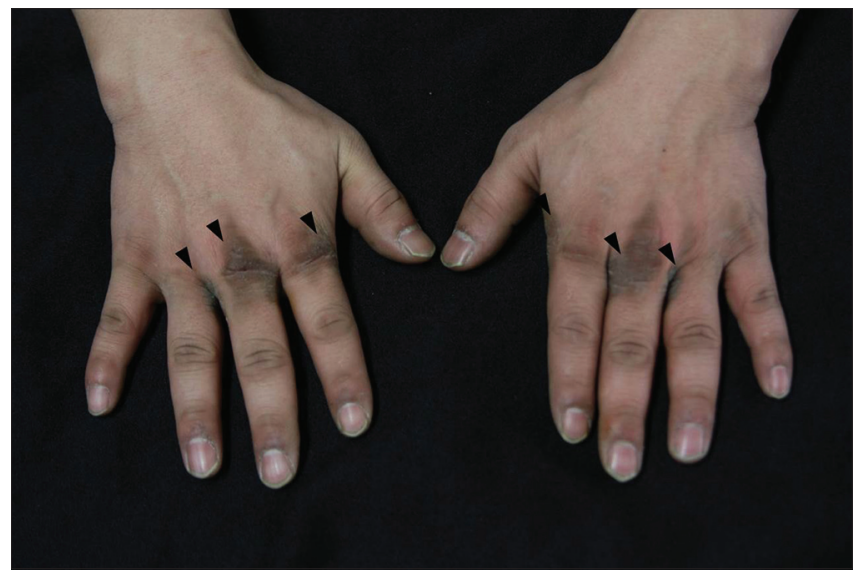

Figure 1: Symmetrical keratoderma localized to the interdigital spaces of the fingers.

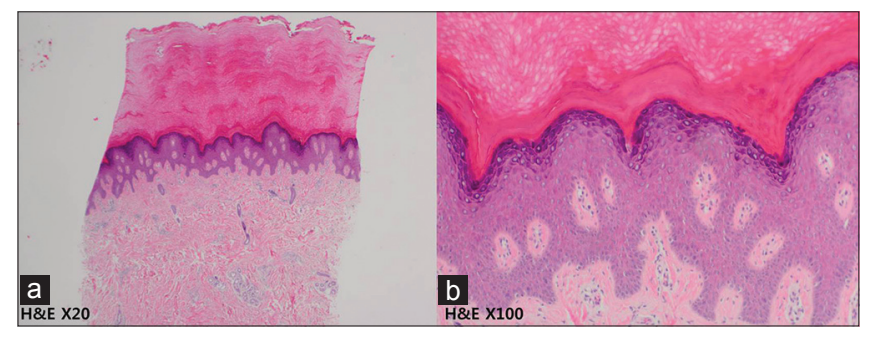

Figure 2: a) Histology revealed compacted orthokeratosis, hypergranulosis with variably elongated epidermis and without significant infiltration of inflammatory cells (H\&E x20). b) Higher magnification revealed prominent acanthosis and papillomatous various of epidermis (H\&E x100).

interdigital spaces of the hand. In 1993, Patrizi et al. reported the case of a 7-year-old female who showed symmetrical lesions of the second, third and fourth interdigital spaces of the hands [3]. In 1995, Di Lernia et al. reported a similar case of a 28-year-old male who showed symmetrical lesions of all the finger interdigital spaces of the hands [4]. In 2004, Raddadi reported the case of a 2-year-old female who showed similar clinical condition of previously reported cases [5]. Because there has been only few cases reported,

\footnotetext{
How to cite this article: Osung K, Hyun C, Joonsoo P. Symmetrical interdigital hyperkeratosis of the hands. Our Dermatol Online. 2018;9(1):84-85 Submission: 18.10.2017; Acceptance: 18.11.2017

DOI:10.7241/ourd.20181.25
} 
the pathogenesis, clinical findings, and treatment of symmetrical interdigital hyperkeratosis. To put the various reports together, the disease occurs mainly from first decade to third decade with no occupational, traumatic, or irritant factor that could affect the lesion [6]. Clinically, it shows keratoderma on the both interdigital spaces of the hands and feet. In majority of cases, the lesions were limited to the fingers and didn't involve the entire hands. Salamon reported a case of symmetrical interdigital hyperkeratosis with scrotal tongue and highly arched palate, but there weren't any accompanied skin lesion or other lesions. Palm was not involved and did not accompany hyperhydrosis. Histological examination shows hyperkeratosis, hypergranulosis, and irregular acanthosis. The lesions responded poorly to the treatment such as topical steroid or keratolytics and this can be helpful to the diagnosis. Among reported cases, 2 cases had family history of the disease and feet were involved in both cases. Other 4 cases including this case were sporadic occurrence and localized exclusively to the hands. Despite the symmetrical interdigital hyperkeratosis is not well known, if appears to involve the foot in congenital type and sporadic type is limited to the hands. This could suggest that interdigital hyperkeratosis could be divided into two subtypes, congenital and sporadic, which show different clinical findings.
Considering the lack of insight and subjective symptom of disease, the prevalence of disease could be higher than it is reported. Evaluation of pathogenesis, specific findings, and treatment of disease with additional case reports is needed.

\section{REFERENCES}

1. Dimitropoulos VA. Dermatofibrosarcoma protuberans. Dermatol Ther. 2008;21:428-32.

2. Kovarik CL, Hsu M-Y, Cockerell CJ. Neurofibromatous changes in dermatofibrosarcoma protuberans: a potential pitfall in the diagnosis of a serious cutaneous soft tissue neoplasm. J Cutan Pathol. 2004;31:492-6.

3. Fiore M, Miceli R, Mussi C, Lo Vullo S, Mariani L, Lozza L, et al. Dermatofibrosarcoma protuberans treated at a single institution: a surgical disease with a high cure rate. J Clin Oncol. 2005;23:7669-75.

4. Heenan PJ. Tumor of the fibrous tissue involving the skin. In: Elder DE, Elenitas R, Johnson BL Jr, eds. Lever's histopathology of the skin. Philadelphia, PA: Lippincott Williams \& Wilkins; 2005:979-1013.

5. Grouls V. Dermatofibrosarcoma protuberans: a variant myxoid. Pathol. 1984;5:226-30.

6. Reimann JD, Fletcher CD. Myxoid dermatofibrosarcoma protuberans: a rare variant analyzed in a series of 23 cases. Am J Surg Pathol. 2007;31:1371-7.

Copyright by Kwon Osung, et al. This is an open access article distributed under the terms of the Creative Commons Attribution License, which permits unrestricted use, distribution, and reproduction in any medium, provided the original author and source are credited.

Source of Support: Nil, Conflict of Interest: None declared. 